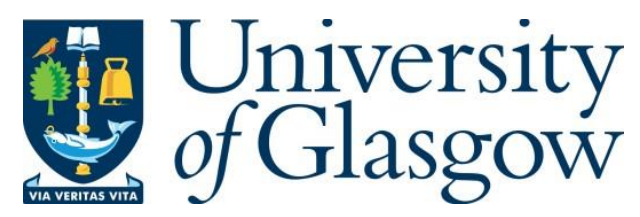

Brunton, E. K., Silveira, C., Rosenberg, J., Schiefer, M. A., Riddell, J. and Nazarpour, K. (2019) Temporal modulation of the response of sensory fibers to paired-pulse stimulation. IEEE Transactions on Neural Systems and Rehabilitation Engineering, 27(9), pp. 1676-1683.

There may be differences between this version and the published version. You are advised to consult the publisher's version if you wish to cite from it.

http://eprints.gla.ac.uk/203625/

Deposited on: 18 November 2019

Enlighten - Research publications by members of the University of Glasgow http://eprints.gla.ac.uk 


\title{
Temporal Modulation of the Response of Sensory Fibers to Paired-Pulse Stimulation
}

\author{
Emma K. Brunton, Member, IEEE, Carolina Silveira, Student Member, IEEE, Joshua Rosenberg, Matthew A. \\ Schiefer, Member, IEEE, John Riddell, and Kianoush Nazarpour, Senior Member, IEEE
}

\begin{abstract}
Multi-channel nerve cuff electrode arrays can provide sensory feedback to prosthesis users. To develop efficacious stimulation protocols an understanding of the impact that spatiotemporal patterned stimulation can have on the response of the sensory fibers is crucial. We used experimental and modelling methods to investigate the response of nerve fibers to pairedpulse stimulation. Nerve cuff electrode arrays were implanted for stimulation of the sciatic nerves of rats and the sensory compound action potentials were recorded from the $L_{4}$ dorsal root. A model of the nerve cuff electrode array and sciatic nerve was also developed. The experimental and modelling results were compared. Experiments showed that it took $8 \mathrm{~ms}$ for the sensory fibers to completely recover from a conditioning stimulus, regardless of the relative position of the electrodes used for stimulation. The results demonstrate that the electrodes on the cuff cannot be considered independent. Additionally, at the stimulus level used here, there is a large overlap in the fibers that were activated by the different electrodes. If a stimulus paradigm considered the electrodes as independent, stimuli from the different electrodes would need to be interleaved, and the intervals between the stimuli should be greater than $8 \mathrm{~ms}$.
\end{abstract}

\section{INTRODUCTION}

A sense of touch is vital when it comes to interacting and experiencing the world around us [1], [2]. In the case of limb difference (loss or absence of limb), mechatronic hands have advanced over the past decades, however, the addition of sensory perception is still in its infancy [2]. Providing prosthetic hand users with sensory perception has been shown not only to greatly improve control of the hand, but also promote a sense of embodiment and reduce phantom limb pain [1], [3]. Substituting sensation with external devices has been shown to help in laboratory settings, however, none of these devices have been widely adopted [2]. Electrical stimulation of the nerves in the residual limb has the potential to provide sensory information from a prosthetic hand [4]-[10].

A number of devices that interface directly with the peripheral nerves have been developed to provide electrode

This work is supported by the UK Engineering and Physical Sciences Research Council (EPSRC) research grants EP/M025977/1, EP/N023080/1 and EP/R004242/1.

Corresponding authors are E. Brunton and K. Nazarpour. Emails: \{emma.brunton,kianoush.nazarpour\}@ newcastle.ac.uk

E. Brunton and C. Silveira are with the School of Engineering, Newcastle University, Newcastle-upon-Tyne, UK.

J. Rosenberg is with the Department of Biomedical Engineering, Case Western Reserve University, Cleveland, Ohio, USA.

M. Schiefer is with the Malcom Randall VA Medical Center, Gainesville, Florida, USA.

J. Riddell is with the Institute of Neuroscience and Psychology, University of Glasgow, UK.

K. Nazarpour is with the School of Engineering and the Institute of Neuroscience, Newcastle University, UK. stimulation [3], [11]. These neural interfaces include intrafascicular electrodes that penetrate the nerves (TIMEs [6], [12], [13], LIFEs [14], [15]) and cuff electrode arrays that wrap around the nerve without penetrating it (Spiral cuffs [1], [16], FINEs [17], [18]). Generally, nerve cuff electrode arrays do not stimulate as selectively as intrafasciular electrode arrays. However, they have been shown to provide a stable interface with the nerve, and to evoke realistic sensory sensations [1].

Testing of multi-channel cuffs in humans has mainly focused on the ability to modulate the perceived sensation by tuning the frequency, amplitude, or pulse width delivered by a single electrode [1], [2], [4], [6]. Spatio-temporal patterns of electrical stimulation delivered from multiple electrodes has the potential to provide patients with different sensations experienced concurrently. In cochlear [19], [20] and retinal implants [21] interactions between electrodes can greatly alter the resultant percept. For example, stimulating the nerve with two electrodes simultaneously results in significant interactions between adjacent electrodes due to the vector summation of their electric fields [20], [22], [23]. As a result, cochlear implants employ strategies that interleave stimuli to avoid electrode interactions [19], [20]. Additionally, even after the electric field applied by an electrode has been removed, the nerve still needs to recover [24]. This can result in changes to the response of nerve fibers to the same stimulus even if stimuli are applied asynchronously. Therefore, it cannot be assumed that asynchronous stimuli will produce independent percepts and stimulus paradigms that consider electrode channel independence will need to carefully consider the timings between sequential stimuli. In stimulus paradigms that move beyond electrode channel independence, interactions between electrodes could be taken advantage of [20]. This is the case in current steering, also known as field shaping, where the electric fields generated by two or more electrodes stimulated simultaneously are combined to target a specific population of fibers within the nerve [16], [25]-[27]. These studies show that knowledge of the spatio-temporal interactions of an electrode array is essential for developing effective patterns of stimulation in a sensory prosthesis.

Spatio-temporal interaction studies to date in peripheral nerves, e.g. the sciatic nerve, have been limited to their effects on motor fibers, as the response of these fibers can be inferred from twitch force [28]-[30] and ankle torque [17], [31] measurements. These studies have shown that both intrafascicular [28], [29], [32] and nerve cuff electrode arrays [16], [17], [33] can be used to selectively stimulate motor fibers from different branches of the sciatic nerve. In addition, they have 
shown that through interleaving multi-site stimulation, fatigueresistant and ripple-free motor responses can be generated [30], [33]. Due to the difficulty of isolating sensory fibers, little work has been done to determine if sensory fibers will behave in the same way. However, we expect that sensory fibers will behave in a similar fashion to motor fibers, albeit with a lower threshold to generate an action potential [1].

Modelling of the electric fields generated in combination with simulations of axon populations are invaluable in the study of spatio-temporal interactions. This is because examination of a large range of parameters would not be feasible to test in clinical or preclinical studies [34]. Models allow for the effects of the location of the electrode contact in relation to fascicles or nodes of Ranvier to be investigated [31], [35]. This would be near impossible to test in-vivo. Models can also provide greater insight into what state both the fast and slow acting voltage-gated sodium channels are in [36].

We examine how spatio-temporally patterned stimulation of the sciatic nerve affects the sensory responses on the $\mathrm{L}_{4}$ dorsal root. We compare the results from laboratory experiments and computer modelling. We characterize the effects of varying both the delay between sequential stimuli, and the spatial location of the electrodes on the compound action potentials (CAPs) generated at $\mathrm{L}_{4}$ dorsal root. We address two questions: (1) whether or not stimulation from different electrodes on the multi-channel cuff could be considered independent; and (2) if the electrodes can not be considered independent, what interstimulus interval is required so that these interactions do not have an effect on the response of the sensory fibers.

\section{Methods}

We describe the experimental and simulation studies used to investigate the spatio-temporal interactions of stimuli that were delivered with a multi-channel cuff electrode array.

\section{A. Animal Preparation}

All procedures were performed under appropriate licences issued by the UK Home Office under the Animals (Scientific Procedures, Act, 1986) and were approved by the Animal Welfare and Ethical Review Board of Newcastle University.

Four Sprague Dawley rats were used in this study weighing from 400 to 475 grams. Anaesthesia was induced in a box with $3 \%$ isoflurane in Oxygen. After anaesthesia was induced, the animal was moved onto a surgical table where anaesthesia was maintained through a mask. To help maintain anaesthetic depth, a subcutaneous injection of meloxicam was given at a dose of $1 \mathrm{mg} / \mathrm{kg}$. Anaesthetic depth was assessed through monitoring of the animal's heart and breathing rates and its responses to noxious toe pinches. Anaesthetic level was adjusted as needed throughout the procedure. Fluids were delivered through a tail vein cannula at $0.2 \mathrm{ml} /$ hour $(20 \mathrm{ml}$ $0.9 \% \mathrm{NaCl}$ and $5 \%$ glucose, with $0.05 \mathrm{ml} \mathrm{KCl}$ ).

An incicision in the skin was made over the $\mathrm{L}_{2}$ to $\mathrm{L}_{6}$ vertebrae (Fig. 1a). Muscle tissue was thoroughly cleared from around the $\mathrm{L}_{6}$ spinous process for placement of the ground electrode. The $\mathrm{L}_{6}$ spinous process was left in place and a tungsten wire was wrapped around it to act as a ground electrode for recordings. The wire was then secured with dental acrylic. To expose the $\mathrm{L}_{4}$ dorsal root a restricted lateralmedial laminectomy was performed. The opening was then covered in saline and gauze to keep the tissue wet while the rest of the surgery was carried out.

A concentric nerve cuff electrode (Microprobes for Lifescience, USA) was implanted on the proximal side of the sciatic nerve following procedures described previously [37], [38]. Briefly, an incision was made in the skin approximately $0.5 \mathrm{~cm}$ caudal and parallel to the right femur. The two planes of the biceps femoris muscle were dissected to expose the sciatic nerve (Fig. 1a). The nerve was freed from the surrounding tissue in preparation for implantation of the cuff electrode array. Two tungsten wire hooks were placed in the tibialis anterior (TA) muscle to monitor electromygraphy (EMG).

The cuff electrode arrays had an inner diameter of $1 \mathrm{~mm}$ with sixteen channels arranged in four rings of four contacts (Fig. 1b). Each ring was separated by $0.75 \mathrm{~mm}$. Each contact was made from $100 \mu \mathrm{m}$ platinum wire and had a surface area of approximately $0.0629 \mathrm{~mm}^{2}$. All other electrodes were made in-house from tungsten insulated wire of $125 \mu \mathrm{m}$ diameter (Advent Research Materials, UK).

After the cuff electrode array was secured with Kwik-Cast (World Precision Instruments, USA), the muscles and skin were closed above the nerve cuff with tissue glue and the gauze and saline were removed from the opening above the spinal cord. The dura was cut to expose all the spinal roots. The $\mathrm{L}_{4}$ dorsal root was identified after locating the $\mathrm{L}_{4}$ dorsal root ganglion. The $\mathrm{L}_{4}$ dorsal root was then separated from the others, lifted and placed across tungsten wire hook electrodes using a glass hook. The tungsten hooks were separated by approximately $1 \mathrm{~mm}$, and connected to form a bipolar pair with an electrode located $2 \mathrm{~mm}$ away (Fig. 1c). The root was placed over three hooks. Only one bipolar channel recorded, if it was not long enough to be placed over the four hooks without stretching. Otherwise, the root was placed over four hooks and two bipolar channels were recorded. The opening was then filled with paraffin oil to insulate the recording electrodes from the surrounding tissue.

\section{B. Neural Recording}

CAPs were recorded using bipolar hook electrodes placed on the $\mathrm{L}_{4}$ dorsal root. While the rat sciatic nerve contains sensory fibers that project to the dorsal root ganglia from $\mathrm{L}_{3}$ to $\mathrm{L}_{6}$, we chose to record from $\mathrm{L}_{4}$ due to space restrictions, and that $98-99 \%$ of sciative nerve neurons project to either $\mathrm{L}_{4}$ or $\mathrm{L}_{5}$ [39]. However, this does means that we may not have been capturing the complete effects of the stimulation. The electroneurographic (ENG) signals were bandpassed filtered between 10 and $5000 \mathrm{~Hz}$, and amplified using a differential amplifier (A-M systems ${ }^{\mathrm{TM}}$, USA). The output from the amplifier was connected to an analogue input of a Cerebus Neural Signal Processor (Blackrock Microsystems, USA) and sampled at a rate of $30 \mathrm{kHz}$.

\section{Neural Stimulation}

Stimuli were delivered to the sciatic nerve through each electrode on the 16-channel cuff electrode array using a Ceres- 
1

2

3

4

5

6

7

8

9

10

11

12

13

14

15

16

17

18

19

20

21

22

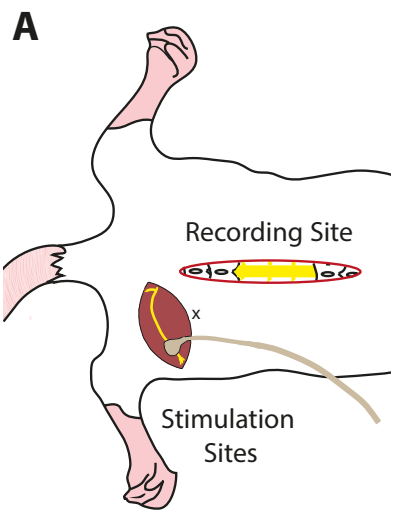

B

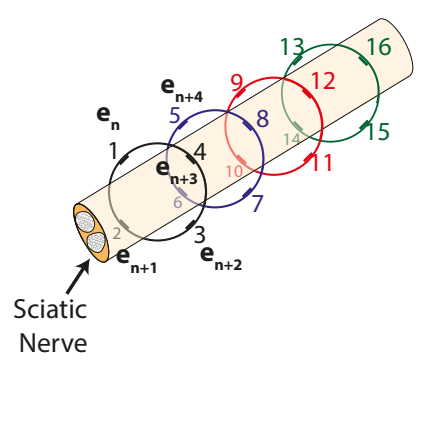

C

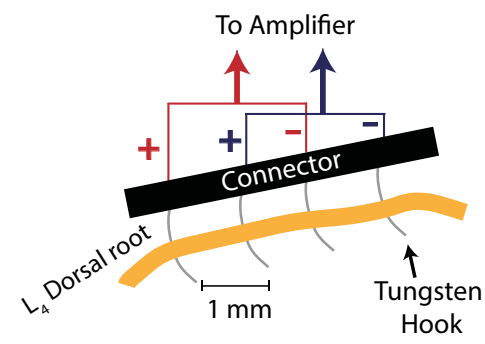

D

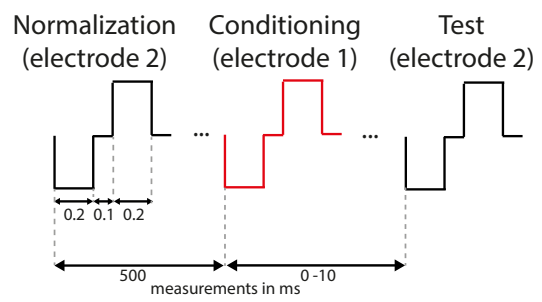

E

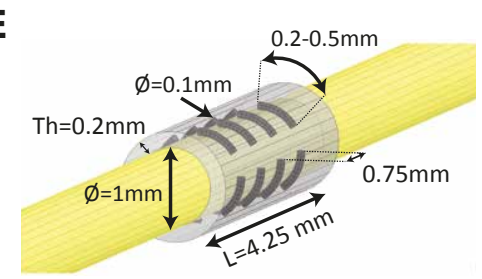

Fig. 1. Experiment Setup: (a) Animal preparation. A multi-channel cuff electrode array was implanted on the sciatic nerve to deliver stimulations, recordings were made from the L4 dorsal root, $\mathrm{x}$ marks the approximate location of the current return before the skin was closed; (b) Illustration of the nerve cuff electrode array arrangement indicating an example of the electrode pair combinations used as described in Table 1; (c) Recordings of compound action potentials were made using hook electrodes placed on the $\mathrm{L}_{4}$ dorsal root; (d) The paired-pulse paradigm used for stimulation; (e) A finite element model of the nerve cuff electrode array and rat sciatic nerve.

tim R96 (Blackrock Microsystems, USA). The experiment was conducted in two parts. First, the threshold current required to elicit a CAP that could be identified on a single-trial basis on an oscilloscope was found. The stimuli used to determine threshold and all subsequent stimulations were monopolar, biphasic, cathodic first, current pulses with a pulse width of $200 \mu \mathrm{s}$ and an inter-pulse-interval of $100 \mu \mathrm{s}$. The current return path was a tungsten wire placed in the skin, above the sciatic nerve. All parameters were kept constant except for the current amplitude. The current amplitude was initially set to $40 \mu \mathrm{A}$ and stepped up or down at intervals of $5 \mu \mathrm{A}$. When close to the threshold current, the step size was reduced to $1 \mu \mathrm{A}$. After finding threshold a current that generated the maximum CAP was found by finding a current that when the amplitude was increased further, no detectable increase in CAP could be seen. The current was then increased beyond this level to ensure that the maximum CAP was recorded. Each electrode was stimulated 10 times at threshold, $120 \%$ of threshold and at a current amplitude that generated the maximum response. The recordings were averaged across the trials. In Animal 2, electrode 14 was broken. This electrode was removed from all animals so that equal comparisons could be made.

For part 2, the nerve was stimulated with a pair of electrodes using current amplitudes of $120 \%$ of threshold. A paired-pulse stimulation paradigm was used where a first "conditioning" pulse was sent from one electrode, $\mathrm{e}_{n},(n=1,2, \ldots, 12)$. A second "test" pulse was then sent from a second electrode that could be in one of five possible locations relative to the first electrode $\left(\mathrm{e}_{n}, \mathrm{e}_{n+1}, \mathrm{e}_{n+2}, \mathrm{e}_{n+3}\right.$ or $\left.\mathrm{e}_{n+4}\right)$ as illustrated in Fig. 1b. These five possible locations are described in more detail in Table 1 and were labelled as: "origin", 90 degrees, 180 degrees, 270 degrees and 0 degrees. In Animal 1, the origin location was not tested.

The time period between the conditioning and test pulses was varied from 0 to $10 \mathrm{~ms}$ in $1 \mathrm{~ms}$ steps. Preceding the conditioning pulse by 0.5 seconds, a single "normalization" pulse, identical to the test pulse was delivered, as illustrated in Fig. 1d. The normalization pulse was used to normalize the CAP to account for any changes in the nerve's responsiveness over time. Each stimulus combination was repeated 10 times.

\section{Analysis of the ENG recordings}

The ENG recordings were analysed offline in MATLAB ${ }^{\mathrm{TM}}$. In the interest of consistency between all animals, only one bipolar channel was used for data analysis. A synchronisation signal from the Cerestim was used to segment the data. The recordings from the 10 repeats for each stimulus combination was then averaged over a period of 0 to $20 \mathrm{~ms}$, where 0 $\mathrm{ms}$ corresponded to the detection of the rising edge of the synchronisation signal. The peak-to-peak response of the CAP was calculated by first finding the minimum and maximum potentials recorded over the time period of 1.5 to $3 \mathrm{~ms}$ after the initiation of the test pulse. The minimum potential was subtracted from the maximum. The time period was chosen to contain the entire CAP and exclude the stimulus artefact.

\section{E. Simulations}

A semi-infinite nerve was modelled with a length of $60 \mathrm{~mm}$ (in the $z$-direction) and simulated using 64 FEMS developed by SimNeurex LLC (Gainesville, FL). The nerve contained 
TABLE I

DESCRIPTION OF THE FIVE POSITIONS THAT THE ELECTRODES THAT DELIVERED THE CONDITIONING AND TEST PULSES COULD BE LOCATED IN.

\begin{tabular}{|c|c|c|c|}
\hline Label & Description & Conditioning & Test \\
\hline origin & The conditioning and test pulses are delivered by the same electrode & $e_{n}$ & $e_{n}$ \\
\hline 90 degrees & $\begin{array}{l}\text { The conditioning and test pulses are delivered by electrodes that are separated by } 90 \text { degrees. They could } \\
\text { be located on the same ring or on adjacent rings. }\end{array}$ & $e_{n}$ & $e_{n+1}$ \\
\hline 180 degrees & $\begin{array}{l}\text { The conditioning and test pulses are delivered by electrodes that are separated by } 180 \text { degrees. They } \\
\text { could be located on the same ring or on adjacent rings. }\end{array}$ & $e_{n}$ & $e_{n+2}$ \\
\hline 270 degrees & $\begin{array}{l}\text { The conditioning and test pulses are delivered by electrodes that are separated by } 270 \text { degrees. They } \\
\text { could be located on the same ring or on adjacent rings. }\end{array}$ & $e_{n}$ & $e_{n+3}$ \\
\hline 0 degrees & $\begin{array}{l}\text { The conditioning and test pulses are delivered by electrodes that are separated by } 0 \text { degrees and are } \\
\text { located on adjacent rings. }\end{array}$ & $e_{n}$ & $e_{n+4}$ \\
\hline
\end{tabular}

two fascicles based on histology obtained from the proximal end of the rat sciatic nerve [40]. The larger fascicle was 0.61 $\mathrm{mm}$ in diameter while the smaller fascicle was $0.35 \mathrm{~mm}$. Both fascicles were modelled as an endoneurium contained within a perineurial sheath that was equal to $3 \%$ of the fascicle diameter [41]. A nerve cuff electrode array was centered on the nerve.

The cuff electrode array was modelled as a silicone sleeve with inner diameter of $1 \mathrm{~mm}$ and $4.25 \mathrm{~mm}$ in length, with a 0.2 $\mathrm{mm}$ thick wall, approximating the array used in experiments. A total of 16 platinum electrodes were included, simuting the used cuff electrode array. Adjacent rows of electrodes were $0.75 \mathrm{~mm}$ apart. The diameter of each electrode was $100 \mu \mathrm{m}$. The arc-length of each electrode was varied from 0.2 to 0.5 $\mathrm{mm}$ in $0.1 \mathrm{~mm}$ steps (Figure 1E). A $1 \mathrm{~mA}$ cathodic current was applied to each electrode independently. The fields generated by multiple electrodes were summed. The nerve-cuff complex was centred in a saline volume measuring $100 \times 100 \times 200$ $\mathrm{mm}^{3}$. The outer borders of the saline were set as sinks. Electrical conductivities of all materials can be found in [18].

Using the DC Conduction solver with a stopping threshold of $0.5 \%$ error and an adaptive mesher, each model required approximately 5-10 minutes and 100,000-200,000 tetrahedra to converge to a solution. The potential (voltage) field within each fascicle was exported to MATLAB. The exported fields were used to linearly interpolate the extracellular potential along axons. Specifically, 1000 axons were randomly positioned within each fascicle. Each axon contained 41 nodes of Ranvier. The diameter of the axons ranged from 4 to $15 \mu \mathrm{m}$ with a bimodal distribution with peaks at 4 and $9 \mu \mathrm{m}$ [42].

We simulated the axons once the extracellular potential was interpolated along the randomly positioned and sized axons. The double cable axon model was used [43]. This model is based on a mammalian motor axon, rather than sensory fibers. For the experiments we used a pulse amplitude that was $120 \%$ of the threshold. For the simulations we assumed that at threshold $10 \%$ of the axons fired an action potential. Thus, we first determined the stimulation threshold for every axon. Stimulation thresholds were then sorted in ascending order. The threshold was determined as the pulse amplitude required to generate an action potential in $10 \%$ of the axons. We then simulated stimulus pulses at $120 \%$ of the threshold. The time delays between each stimulus pulse was stepped from 0 to $10 \mathrm{~ms}$ in steps of $1 \mathrm{~ms}$. This sweep was repeated for every combination of the 16 electrodes at the five possible angles, within the 4 families of electrode lengths, and for every axon, totalling 1024 possible combinations for each axon. Additionally, axons were simulated with only one active electrode to isolate the timing characteristics of an action potential produced by that electrode during post-processing.

All simulations were run at the Ohio Supercomputer Center [44]. Each combination of parameters was run in parallel on a 28-core machine and required three hours of wall-time per electrode combination for a total of 768 hours of computation time. Symmetry of the electrodes within the cuff array allowed us to eliminate half of the simulations, considering only the combinations of electrodes in the first and second row with any of the other electrodes. To reduce the amount of storage from the simulation results, the voltage at each time point was only stored for every fifth node of Ranvier.

\section{F. Analysis of Simulated Data}

The output from the simulations comprised the voltage against time data for every axon and electrode parameter combination. The voltage values represented the extracellular voltage at every 5 th node of Ranvier in $5 \mu$ s time steps for the duration of the stimulus. In the case where the pulse amplitude was below threshold, a zero was stored to indicate that no action potentials would have occurred. In the case where an action potential was generated in an axon (from here on called an active axon), the peak voltage was stored. This analysis was repeated for every axon for each of the electrode combinations. To investigate the effect of a conditioning pulse on the response of the fibers, we counted the number of active axons in response to the second (test) pulse and compared this to the number of active axons when a single pulse was delivered on the same electrode, i.e. the one that delivered the test pulse, without a condition pulse. The ratio between active axons with and without a conditioning pulse was calculated for each time delay.

\section{RESUlTS}

The effect of the spatio-temporal interactions between electrodes on the recovery of the response of sensory fibers was investigated both experimentally and with modelling. In all four animals the CAPs were recorded at the $\mathrm{L}_{4}$ dorsal root in response to stimulation of the sciatic nerve. A paired-pulse paradigm was used to investigate the recovery of the response of sensory fibers, where 11 different temporal spacings and 5 different spatial positions were examined in all animals except Animal 1, where the origin spatial condition was not tested. 
1

2

3

4

5

6

7

8

9

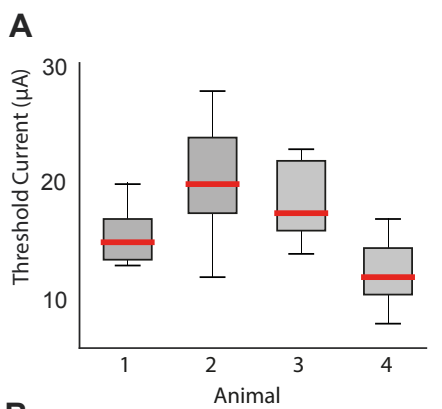

B
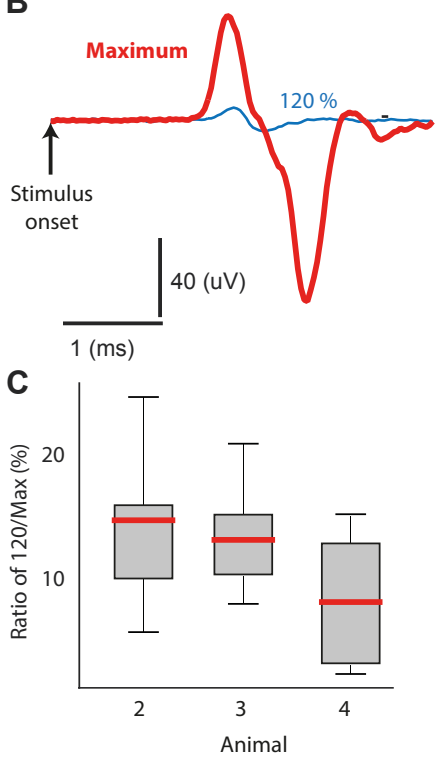

Fig. 2. (a) Boxplot indicating the range of thresholds found in each animal. (b)An example of recordings made on electrode 2 in Animal 2 showing the compound action potential recorded at $120 \%$ and one recorded at maximum. (c) Box plot of the distribution of the ratio of the peak-to-peak amplitudes of the CAP recorded at $120 \%$ of threshold to the maximum peak-to-peak amplitude of the CAP recorded. Shown for Animals 2-4, the maximum potential was not recorded in Animal 1. On the boxpolots the red line in the centre indicates the median, the bottom and top edges of the box indicate the 25 th and 75 th percentiles, the whiskers extend to the most extreme data points, ( $n=16$ for Animals 1, 3 and 4, $n=15$ for Animal 2).

\section{A. Thresholds to elicit CAPs}

The threshold to elicit a detectable CAP on a single trial was measured for each of the 16 channels of the multi-channel cuff electrode array in all four animals. A box plot illustrating the distribution of thresholds for each animal is shown in Fig. 2a. At threshold, where a CAP was detected on the $\mathrm{L}_{4}$ dorsal root, there was no twitch detected in the leg, or observable EMG recorded on the TA muscle. The thresholds to elicit a CAP were also lower on average than the thresholds required to elicit a muscle movement using the same cuffs in previous work [22]. As would be expected as sensory fibers have been found to have lower thresholds than motor fibers [1]. At 120\% of threshold, EMG was only detected on Electrode 1 in Animal 1 and Electrode 15 in Animal 2. No EMG was detected on either Animal 3 or 4 with a pulse amplitude of $120 \%$ of threshold.

To ensure that at $120 \%$ of threshold the whole nerve was not stimulated, we compared the CAPs recorded at $120 \%$ of thresholds to the maximum CAPs generated in Animals 2-4.
An example of a maximum response and a $120 \%$ response is presented in Fig. 2b. The maximum response was not recorded in Animal 1. The ratio of the peak-to-peak of the CAP recorded at $120 \%$ to the peak-to-peak of the CAP recorded at maximum current amplitude was calculated in percent and is plotted for each animal in Fig. 2c. The mean ratios were 17, 13 and 8 percent for Animals 2, 3, and 4 respectively.

\section{B. Temporal separation of pulses}

The ENG of the $\mathrm{L}_{4}$ dorsal root was recorded in response to biphasic cathodic first pulses applied to the sciatic nerve. An example of the ENG recordings made is shown in Fig. 3A, where the interval between the conditioning and the test pulse were varied. In this representative recording, when the inter stimulus interval was 1 or $2 \mathrm{~ms}$, the recorded CAP does not differ from the recordings made at other time intervals. This indicates that no detectable CAP was generated at these two intervals and the peak at $1 \mathrm{~ms}$ is due to the conditioning pulse.

The peak-to-peak value of the compound action potential was measured. The box plot in Fig. 3b shows the representative results for one representative animal, Animal 3. For each animal the mean peak-to-peak of the CAP recovered to within $90 \%$ of the normal response after about $8 \mathrm{~ms}$. Results from all animals are presented in the supplementary material.

\section{Spatial separation of pulses}

The relative position of the two electrodes used to deliver the stimuli was varied in addition to the interval. The two electrodes could have five positions relative to each other: origin, 90 degrees, 180 degrees, 270 degrees or 0 degrees. In Fig. 3, the data has been pooled into groups based on the relative position of the electrodes to each other and the mean \pm the standard error plotted for each stimulus interval. In all animals regardless of the electrode positions a similar trend was seen. The CAP generated by two electrodes that delivered stimuli simultaneously was much larger than that generated by a stimulus delivered by a single electrode. The peaks of the CAPs measured in response to the test pulse delivered 1 to $7 \mathrm{~ms}$ after the conditioning pulse are reduced. Representative results for Animal 3 are shown in Fig. 3c. Results from other animals are in Supplementary Materials.

\section{Comparison of the experiment to the simulation results}

Figure $3 \mathrm{~d}$ shows that the results of the finite element study were similar to the results from the experiments. There is, however, one noticeable difference. In the model, the fibers recovered faster than in the experiments. The model showed that the fibers completely recovered their response after about $5 \mathrm{~ms}$; almost half the time seen in the experiments. All electrode arc-lengths tested showed similar results.

Comparing the simulation results in Fig. $3 \mathrm{~d}$ to the results in Fig. 3c, we observe that at 1-2 ms there is a larger reduction in the response of the nerve fibers in the model than the experiment. However, this is most likely due to how this was measured in the experiments compared to the model. In the experiments, the peak-to-peak value of the signal was 

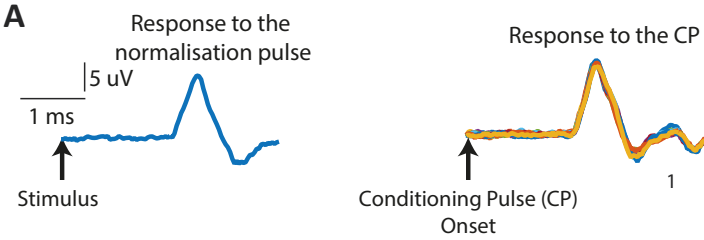

C
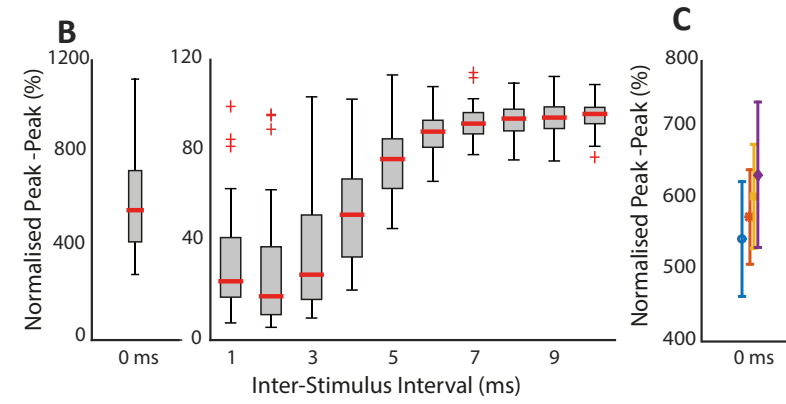
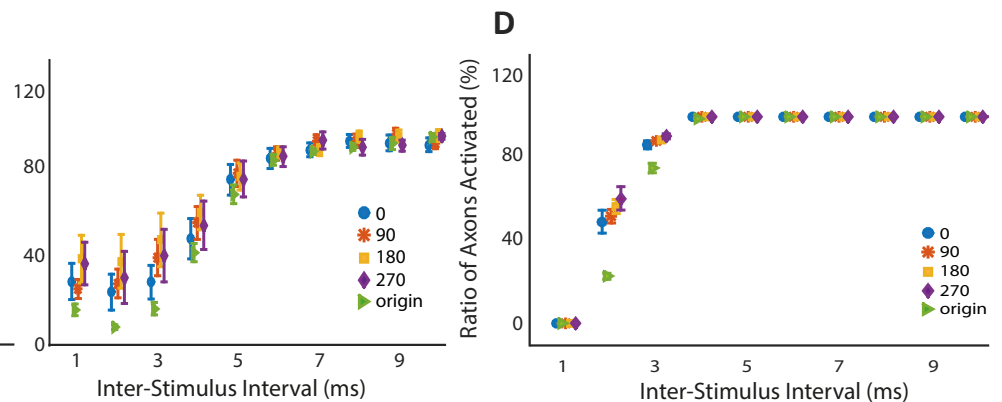

Fig. 3. (a) Recordings of the compound action potentials from the $\mathrm{L}_{4}$ dorsal root. The pulse shows the response of the root to the test pulse without a conditioning pulse. The right hand figure shows the response of the nerve to the conditioning and test pulses, where different intervals between the two pulses were used. This is an example from Animal 2 when the conditioning pulse is delivered by electrode 1 and the test pulse is delivered through electrode 3 . These electrodes were at 180 degrees from each other. (b) Box plots showing the normalized peak-to-peak for Animal 3, where the red line in the centre indicates the median, the bottom and top edges of the box indicate the 25 th and 75 th percentiles, the whiskers extend to the most extreme data points, and the red crosses indicates an outliers. ( $n=55$ for each time interval). The interval of zero milliseconds corresponds to current being delivered through the two electrodes simultaneously. (c) The mean \pm the standard deviation of the normalized peak-to-peak for each electrode spatial position for Animal 3 versus the interval ( $n=11$ for each group at each time interval). The interval of $0 \mathrm{~ms}$ corresponds to current being delivered through the two electrodes simultaneously. (d) The results from finite element model showing the mean \pm the standard deviation of the ratio of axons activated versus the interval. All experimental results were collected with a current amplitude that was $120 \%$ of the threshold for a given electrode. All simulations results used a current amplitude that was $120 \%$ of threshold assuming a threshold pulse generated an action potential in $10 \%$ of the axons.

calculated and normalised, whereas in the model, the percent of axons that generated an action potential can be directly determined. In some cases in the experiment at the 1 and $2 \mathrm{~ms}$ inter-stimulus-intervals, the nerve was still responding to the conditioning pulse, thus the response of the nerve to the testpulse would be overestimated. An example of this can be seen in Fig. 3a. At 1 and $2 \mathrm{~ms}$, all inter-stimulus-intervals traces follow a similar trajectory, indicating the neural response at this time point is due to the conditioning pulse.

\section{Discussion}

We measured the response of sensory fibers to a pairedpulse paradigm, and associated them with spatio-temporal interactions between electrodes. While we only recorded the neural response from $\mathrm{L}_{4}$ and thus were not capturing all the effects of the stimulations applied to the sciatic, inferences can still be made. A finite element model was used to further elucidate what was happening in the nerve. Experimental results showed that regardless of the relative position of the electrodes, the peak-to-peak of the CAP was reduced when a conditioning pulse was delivered less than $8 \mathrm{~ms}$ before the test pulse. When the test and the conditioning pulse were delivered by the same electrode, the largest reduction in the peak-to-peak of the CAP was observed. This was in line with the simulation results. The fibers in the simulation recovered faster than in the experiments; $5 \mathrm{~ms}$ compared to $8 \mathrm{~ms}$. This is most likely due to differences in ion channel dynamics, as the simulation was not developed to exactly model the experiments. In addition to ion channel properties such as maximum conductances, Nernst potentials, gating time constants; ion channel dynamics can be affected by membrane voltage [45] and temperature [46].
The thresholds found here to generate a detectable sensory CAP were on average lower than those found previously to generate a visually detectable muscle twitch [22]. This is in agreement with studies in humans, where sensory percepts are produced before muscle activity is recorded [1]. The maximum current used throughout the experiments was $200 \mu \mathrm{A}$, this corresponds to a $k$-value of 0.4 for the electrodes used, well below the safe threshold of 1.5, as suggested by Shannon [47].

\section{A. Selectivity of Electrodes}

Interactions varied as a function of temporal spacing between the two pulses. The relative position of the two electrodes used to deliver the stimuli had little influence on the response of the sensory fibers to the delivered stimuli. Therefore, regardless of the relative position of the two stimulating electrodes, the stimulations were generating action potentials in an overlapping subset of axons.

One explanation for the selectivity of the stimuli from different electrodes being so poor comes via a study by Leventahl et al. [35]. Using finite element modelling and experiments, Leventhal et al. [35] demonstrated that at low current levels, selectivity can be reduced in comparison to higher currents due to the same group of large diameter fibers being recruited first by different electrodes [35]. At higher currents, they found that smaller diameter axons closer to the individual electrodes were recruited, increasing selectivity up to a point where the the fields from the different electrodes begin to overlap greatly, and selectivity decreases. While, we did not test different current levels, our results suggest that all the electrodes tested appear to be recruiting the same small subset of fibers. This indicates that the current 
amplitude used within our study may be below that required for selective stimulation of smaller fibers. Furthermore, the study by Leventhal et al. [35] also showed that selectivity of electrodes could be improved by aligning them with the different fascicles. Given the difficulty of aligning the nerve cuff electrode arrays with fascicles during surgery, in both our finite element modelling and animal studies, we did not align the electrodes with any of the fascicles. Therefore, it is unlikely that our nerve cuff electrode arrays would be in the optimal position for selectivity. We measured the CAP in response to different stimuli. It is not known if the measured CAP would correspond to a percept in the animal or, if it did, if stimuli delivered by the different electrodes would be identified as different perceptions.

\section{B. Implications for Sensory Feedback Prostheses}

For a sensory feedback prosthesis, providing patients with information about discrete events may be more beneficial than supplying the patient with information continuously [48]. If this discrete event-driven sensory feedback control policy was to be implemented in an invasive prosthesis, then different electrodes on a multi-channel cuff array could be used to signify different events. Such stimulus paradigms would need to make sure the inter-stimulus interval was at least $8 \mathrm{~ms}$ apart to reduce the propensity for spatio-temporal interactions between electrodes to influence the resultant perceptions. Although, the $8 \mathrm{~ms}$ interval here corresponds only to the recovery of the sensory fibers, research is needed to determine the shortest time delay at which humans can detect two different stimuli.

\section{Limitations}

The results of this study are limited to one stimulus amplitude, delivered from electrodes that are relatively close (less than $1 \mathrm{~mm}$ apart) on a cuff. Both stimulation amplitude and position will effect the selectivity of the stimulation [35]. Increasing the distance between the rings on the cuffs, may increase selectivity. Secondly, the pulse amplitude was chosen so that we could visual detect a CAP on every trial. However, decreasing the pulse amplitude closer to threshold would likely also alter the selectivity. Both of these factors may also influence the time it takes for the nerve fibers to recover from the application of the pulse. Further work is needed to see what effect these two factors would have. Thus, the stated recovery time of $8 \mathrm{~ms}$ here may significantly differ if the geometry of the cuff or the pulse amplitude was changed.

\section{CONCLUSION}

For an limb prosthesis to deliver complex sensory information, spatio-temporally patterned stimuli can be used. The results of this study demonstrate that spatio-temporal interactions need to be carefully considered in the design of efficacious stimulation protocols. Stimuli from different electrodes on the multi-channel cuff tested here could not be considered as independent, regardless of their relative positions on the cuff. If a stimulus paradigm considers the electrodes as producing independent percepts, the stimuli will need to be interleaved to reduce the likelihood of electrode interactions affecting the resultant percepts. This study highlights the need for further neuroscience and modelling studies to help elucidate the influence that different stimulus paradigms would have on the resultant percepts experienced by a person.

\section{Data Access Statement}

Data supporting this publication is available under an 'Open Data Commons Open Database License'. Additional metadata are available at: http://dx.doi.org/10.17634/154300-105

\section{CONFLiCt OF INTEREST}

Matthew Schiefer is the owner of SimNeurex LLC.

\section{REFERENCES}

[1] D. W. Tan, M. A. Schiefer, M. W. Keith, J. R. Anderson, J. Tyler, and D. J. Tyler, "A neural interface provides long-term stable natural touch perception," Science Translational Medicine, vol. 6, no. 257, p. 257ra138, Oct 2014.

[2] D. J. Tyler, "Neural interfaces for somatosensory feedback: bringing life to a prosthesis," Current opinion in neurology, vol. 28, no. 6, Dec 2015.

[3] X. Navarro, T. B. Krueger, N. Lago, S. Micera, T. Stieglitz, and P. Dario, "A critical review of interfaces with the peripheral nervous system for the control of neuroprostheses and hybrid bionic systems," Journal of the Peripheral Nervous System., vol. 10, no. 3, pp. 229-258, Oct 2005.

[4] R. Ackerley, H. Backlund Wasling, M. Ortiz-Catalan, R. Brnemark, and J. Wessberg, "Case studies in neuroscience: Sensations elicited and discrimination ability from nerve cuff stimulation in an amputee over time," Journal of Neurophysiology, vol. 120, no. 1, pp. 291-295, Jul 2018.

[5] G. S. Dhillon and K. W. Horch, "Direct neural sensory feedback and control of a prosthetic arm," IEEE Transactions on Neural Systems and Rehabilitation Engineering, vol. 13, no. 4, pp. 468-472, Dec 2005.

[6] G. Valle, F. M. Petrini, I. Strauss, F. Iberite, E. D’Anna, G. Granata, M. Controzzi, C. Cirpriani, T. Stieflitz, P. M. Rossini, A. Mazzoni, S. Raspopovic, and S. Micera, "Comparison of linear frequency and amplitude modulation for intraneural sensory feedback in bidirectional hand prostheses," Scientific Reports, vol. 8, no. 1, p. 16666, Nov 2018.

[7] D. M. Page, J. A. George, D. T. Kluger, C. Duncan, S. Wendelken, T. Davis, D. T. Hutchinson, and G. A. Clark, "Motor control and sensory feedback enhance prosthesis embodiment and reduce phantom pain after long-term hand amputation," Frontiers in Human Neuroscience, vol. 12, no. 352, Sep 2018.

[8] M. Schiefer, D. Tan, S. M. Sidek, and D. J. Tyler, "Sensory feedback by peripheral nerve stimulation improves task performance in individuals with upper limb loss using a myoelectric prosthesis," Journal of Neural Engineering, vol. 13, no. 1, p. 016001, Dec 2016.

[9] S. Raspopovic, M. Capogrosso, F. M. Petrini, M. Bonizzato, J. Rigosa, G. Di Pino, J. Carpaneto, M. Controzzi, T. Boretius, E. Fernandez, G. Granata, C. M. Oddo, L. Citi, A. L. Ciancio, C. Cipriani, M. C. Carrozza, W. Jensen, E. Guglielmelli, T. Stieglitz, P. M. Rossini, and S. Micera, "Restoring natural sensory feedback in real-time bidirectional hand prostheses," Science Translational Medicine, vol. 6, no. 222, pp. 222ra19-222ra19, Feb 2014.

[10] C. M. Oddo, S. Raspopovic, F. Artoni, A. Mazzoni, G. Spigler, F. Petrini, F. Giambattistelli, F. Vecchio, F. Miraglia, L. Zollo, G. Di Pino, D. Camboni, M. C. Carrozza, E. Guglielmelli, P. M. Rossini, U. Faraguna, and S. Micera, "Intraneural stimulation elicits discrimination of textural features by artificial fingertip in intact and amputee humans," eLife, vol. 5, p. e09148, mar 2016.

[11] W. L. C. Rutten, "Selective electrical interfaces with the nervous system," Annual Reviews of Biomedical Engineering, vol. 4, pp. 407452, Mar 2002.

[12] J. Badia, T. Boretius, D. Andreu, C. Azevedo-Coste, T. Stieglitz, and X. Navarro, "Comparative analysis of transverse intrafascicular multichannel, longitudinal intrafascicular and multipolar cuff electrodes for the selective stimulation of nerve fascicles," Journal of Neural Engineering, vol. 8, no. 3, p. 036023, May 2011. 
[13] T. Boretius, J. Badia, A. Pascual-Font, M. Schuettler, X. Navarro, K. Yoshida, and T. Stieglitz, "A transverse intrafascicular multichannel electrode (TIME) to interface with the peripheral nerve," Biosensors \& Bioelectronics, vol. 26, pp. 62-69, May 2010.

[14] L. Citi, J. Carpaneto, K. Yoshida, K.-P. Hoffmann, K. P. Koch, P. Dario, and S. Micera, "On the use of wavelet denoising and psike sorting techniques to process electroneurographic signals recorded using intraneural electrodes," Journal of Neuroscience Methods, vol. 172, pp. 294-302, Jul 2008.

[15] S. M. Lawrence, G. S. Dhillon, W. Jensen, K. Yoshida, and K. W. Horch, "Chronic multichannel neural recordings from soft regenerative microchannel electrodes during gait," IEEE Transactions on Neural Systems and Rehabilitation Engneering, vol. 12, pp. 345-348, Sep 2004.

[16] K. H. Polasek, H. A. Hoyen, M. W. Keith, R. F. Kirsch, and D. J. Tyler, "Stimulation stability and selectivity of chronically implanted multicontact nerve cuff electrodes in the human upper extremity," IEEE Transactions on Neural Systems and Rehabilitation Engineering, vol. 17, no. 5, pp. 428-437, Sep 2009.

[17] D. J. Tyler and D. M. Durand, "Functionally selective peripheral nerve stimulation with a flat interface nerve electrode," IEEE Transactions on Neural Systems and Rehabilitation Engineering, vol. 10, no. 4, pp. 294-303, Dec 2002.

[18] M. A. Schiefer, R. J. Triolo, and D. J. Tyler, "A model of selective activation of the femoral nerve with a flat interface nerve electrode for a lower extremity neuroprosthesis," IEEE Transactions on Neural Systems and Rehabilitation Engineering, vol. 16, no. 2, pp. 195-204, Apr 2008.

[19] B. S. Wilson, C. C. Finley, D. T. Lawson, R. D. Wolford, D. K. Eddington, and W. M. Rabinowitz, "Better speech recognition with cochlear implants," Nature, vol. 352, pp. 236-238, Jul 1991.

[20] J. A. Hokanson, R. A. Gaunt, and D. J. Weber, "Effects of synchronous electrode pulses on neural recruitment during multichannel microstimulation," Scientific Reports, vol. 8, p. 13067, Aug 2018.

[21] R. Cicione, J. B. Fallon, G. D. Rathbone, C. E. Williams, and M. N. Shivdasani, "Spatiotemporal interactions in the visual cortex following paired electrical stimulation of the retina," Investigative Ophthalmology and Visual Science, vol. 55, no. 12, pp. 7726-7738, Nov 2014.

[22] E. Brunton, C. W. Blau, and K. Nazarpour, "Multichannel cuff electrodes for peripheral nerve stimulation and recording," in 2016 IEEE International Conference on Systems, Man, and Cybernetics (SMC), Oct 2016, pp. $003223-003227$.

[23] C. Wang, E. Brunton, S. Haghgooie, K. Cassells, A. Lowery, and R. Rajan, "Characteristics of electrode impedance and stimulation efficacy of a chronic cortical implant using novel annulus electrodes in rat motor cortex," Journal of Neural Engineering, vol. 10, no. 4, p. 046010, Jul 2013.

[24] J. L. Parker, N. H. Shariati, and D. M. Karantonis, "Electrically evoked compound action potential recording in peripheral nerves," Bioelectronics in Medicine, vol. 1, no. 1, pp. 71-83, Jan 2018.

[25] J. D. Sweeney, D. A. Ksienski, and J. T. Mortimer, "A nerve cuff technique for selective excitation of peripheral nerve trunk regions," IEEE Transactions on Biomedical Engineering, vol. 37, no. 7, pp. 706715, July 1990.

[26] B. H. Bonham and L. M. Litvak, "Current focusing and steering: Modeling, physiology, and psychophysics," Hearing Research, vol. 242, no. 1, pp. 141-153, Apr 2008.

[27] J. Ballestero, M. Recugnat, J. Laudanski, K. E. Smith, D. J. Jagger, D. Gnansia, and D. McAlpine, "Reducing current spread by use of a novel pulse shape for electrical stimulation of the auditory nerve," Trends in Hearing, vol. 19, p. 2331216515619763 , Dec 2015.

[28] D. McDonnall, G. A. Clark, and R. A. Normann, "Selective motor unit recruitment via intrafascicular multielectrode stimulation," Canadian Journal of Physiology and Pharmacology, vol. 82, no. 8-9, pp. 599609, Aug-Sep 2004.

[29] K. Yoshida and K. Horch, "Selective stimulation of peripheral nerve fibers using dual intrafascicular electrodes," IEEE Transactions on Biomedical Engineering, vol. 40, no. 5, pp. 492-494, May 1993.

[30] D. McDonnall, G. A. Clark, and R. A. Normann, "Interleaved, multisite electrical stimulation of cat sciatic nerve produces fatigue-resistant, ripple-free motor responses," IEEE Transactions on Neural Systems and Rehabilitation Engineering, vol. 12, no. 2, pp. 208-215, Jun 2004.

[31] W. M. Grill and J. T. Mortimer, "Inversion of the current-distance relationship by transient depolarization," IEEE Transactions on Biomedical Engineering, vol. 44, no. 1, pp. 1-9, Jan 1997.

[32] W. L. C. Rutten, H. J. van Wier, and J. H. M. Put, "Sensitivity and selectivity of intraneural stimulation using a silicon electrode array," IEEE Transactions on Biomedical Engineering, vol. 38, no. 2, pp. 192198, Feb 1991.
[33] L. E. Fisher, D. J. Tyler, and R. J. Triolo, "Optimization of selective stimulation parameters for multi-contact electrodes," Journal of NeuroEngineering and Rehabilitation, vol. 10, no. 1, p. 25, Feb 2013.

[34] N. A. Pelot, B. J. Thio, and W. M. Grill, "Modeling current sources for neural stimulation in comsol," Frontiers in Computational Neuroscience, vol. 12, p. 40, Jun 2018.

[35] D. K. Leventhal and D. M. Durand, "Subfascicle stimulation selectivity with the flat interface nerve electrode," Annals of Biomedical Engineering, vol. 31, no. 6, pp. 643-652, Jun 2003.

[36] W. M. Grill and J. T. Mortimer, "Stimulus waveforms for selective neural stimulation," IEEE Engineering in Medicine and Biology Magazine, vol. 14, no. 4, pp. 375-385, Jul 1995.

[37] E. Brunton, C. Blau, C. Silveira, and K. Nazarpour, "Identification of sensory information in mixed nerves using multi-channel cuff electrodes for closed loop neural prostheses," in 8th International IEEE/EMBS Conference on Neural Engineering (NER), May 2017, pp. 391-394.

[38] E. Brunton, C. W. Blau, C. Silveira, , and K. Nazarpour, "Separability of neural responses to standardised mechanical stimulation of limbs," Scientific Reports., vol. 7, p. 11138, Sep 2017.

[39] J. E. Swett, Y. Torigoe, V. R. Elie, C. M. Bourassa, and P. G. Miller, "Sensory neurons of the rat sciatic nerve," Experimental Neurology, vol. 114, no. 1, pp. 82 - 103, 1991.

[40] C. Silveira, E. Brunton, S. Spendiff, and K. Nazarpour, "Influence of nerve cuff channel count and implantation site on the separability of afferent eng," Journal of Neural Engineering, vol. 15, no. 4, p. 046004, Aug 2018. [Online]. Available: http://stacks.iop.org/1741$2552 / 15 / \mathrm{i}=4 / \mathrm{a}=046004$

[41] Y. Grinberg, M. A. Schiefer, D. J. Tyler, and K. J. Gustafson, "Fascicular perineurium thickness, size, and position affect model predictions of neural excitation," IEEE Transactions on Neural Systems and Rehabilitation Engineering, vol. 16, no. 6, pp. 572-581, Dec 2008.

[42] H. S. D. Garven, F. W. Gairns, and G. Smith, "The nerve fibre populations of the nerves of the leg in chronic occlusive arterial disease in man," Scottish Medical Journal, vol. 7, no. 6, pp. 250-265, Jun 1962.

[43] C. C. McIntyre, A. G. Richardson, and W. M. Grill, "Modeling the excitability of mammalian nerve fibers: Influence of afterpotentials on the recovery cycle," Journal of Neurophysiology, vol. 87, no. 2, pp. 995-1006, Feb 2002.

[44] Ohio Supercomputer Center. (2016) Owens Supercomputer. Columbus, OH: Ohio Supercomputer Center. [Online]. Available: http://osc.edu/ark:19495/hpc6h5b1

[45] M. C. Kiernan and H. Bostock, "Effects of membrane polarization and ischaemia on the excitability properties of human motor axons," Brain, vol. 123, no. 12, pp. 2542-2551, Dec 2000.

[46] K. Lowitzsch, H. C. Hopf, and J. Galland, "Changes of sensory conduction velocity and refractory periods with decreasing tissue temperature in man," Journal of Neurology, vol. 216, no. 3, pp. 181-188, Sep 1977.

[47] R. V. Shannon, "A model of safe levels for electrical stimulation," IEEE Transactions on Biomedical Engineering, vol. 39, no. 4, pp. 424-426, Apr 1992.

[48] C. Cipriani, J. L. Segil, F. Clemente, R. F. ff. Weir, and B. Edin, "Humans can integrate feedback of discrete events in their sensorimotor control of a robotic hand," Experimental Brain Research, vol. 232, no. 11, pp. 3421-3429, Nov 2014. 


\section{Supplementary Material}

Emma K. Brunton, Carolina Silveira, Joshua Rosenberg, Matthew A. Schiefer, John Riddell and Kianoush Nazarpour

June 6, 2019

\section{Overview}

This document provides the supplementary material to support the manuscript entitled "Temporal Modulation of the Response of Sensory Fibers to PairedPulse Stimulation" submitted to IEEE Transactions on Neural Systems and Rehabilitation Engineering. 


\subsection{Temporal Spacing Between Pulses}

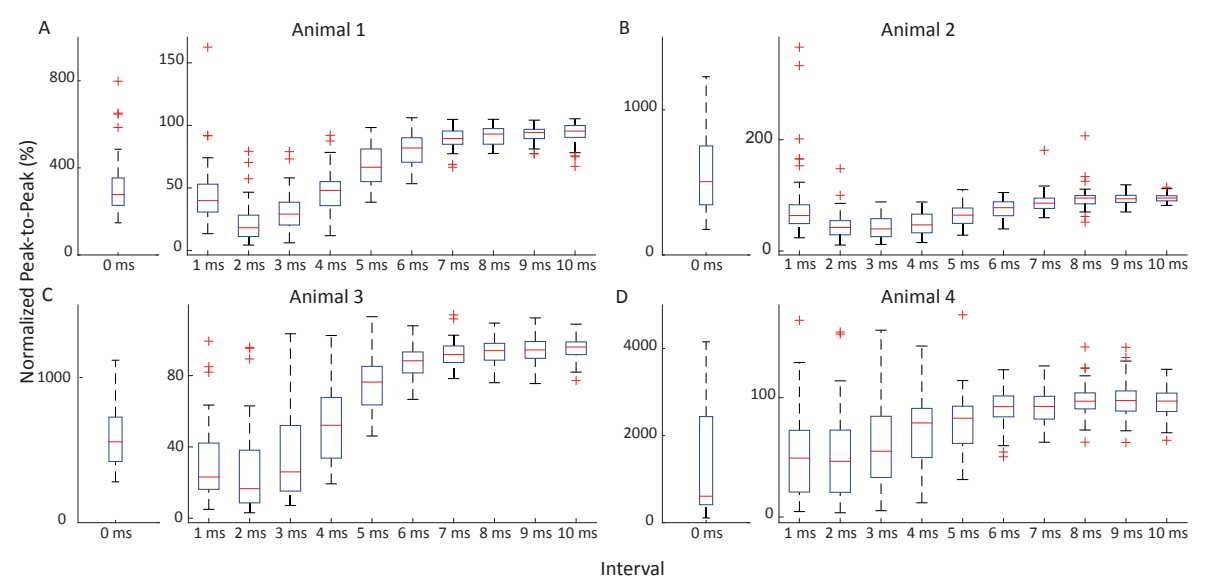

Figure 1: Box plots showing the normalized peak-to-peak value of the compound action potential as the interval between the conditioning and the test pulse is increased. Results are shown for all four Animals:(a) Animal 1, (b) Animal 2, (c) Animal 3 and (d) Animal 4. The red line in the center indicates the median, the bottom and top edges of the box indicate the 25 th and 75 th percentiles, the whiskers extend to the most extreme data points, and the red crosses indicate outliers. ( $n=55$ for each time interval in each animal). This illustrates that similar results were obtained for the same experiment in four different animals on four different days. 


\subsection{Circumferential Spacing Between Electrodes}
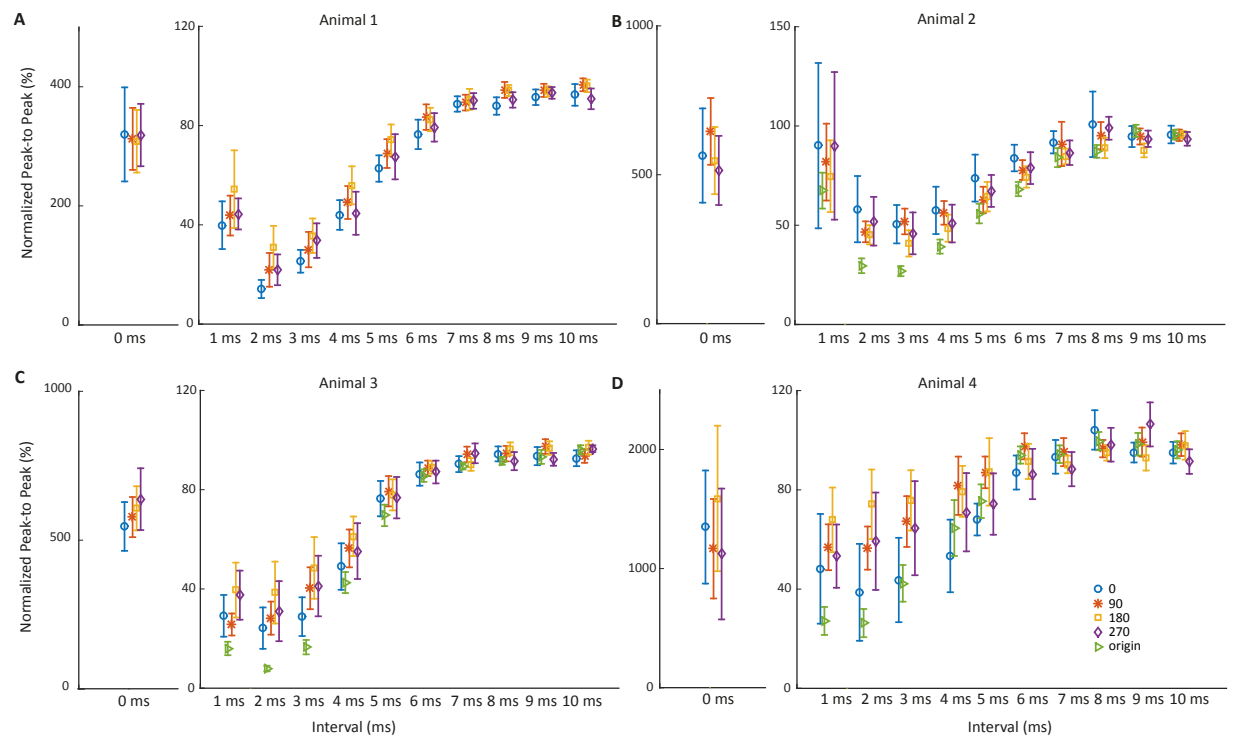

Figure 2: The mean \pm the standard deviation of the normalized peak to peak for each electrode circumferential position. Results are shown for all four Animals:(a) Animal 1, (b) Animal 2, (c) Animal 3 and (d) Animal 4.( $n=11$ for each time interval for each position in each animal). This illustrates that similar results were obtained for the same experiment in four different animals on four different days. 